\title{
Thermal Protective Properties of Aerogel-coated Kevlar Woven Fabrics
}

\author{
Mohanapriya Venkataraman*, Xiaoman Xiong, Jana Novotná \\ Marie Kašparová, Rajesh Mishra, Jiří Militký \\ Technical University of Liberec, Faculty of Textile Engineering, Department of Material Engineering \\ Liberec 46117, Czech Republic
}

\begin{abstract}
In this work, silica aerogels were incorporated with high-performance Kevlar fabrics by coating to deal with heat transfer problems under a severe environment. Thermal protective properties of the prepared fabrics were evaluated by using a laser system coupled with a thermometer which records the back-surface temperature of the fabric under laser radiation. The effect of aerogel content as well as pixel time used for laser radiation on thermal protection was investigated and discussed. Meanwhile, thermal properties of the fabrics under ambient temperature were tested with a thermal camera, thermal insulation values of the fabrics with different coatings were compared and analyzed. Moreover, fly ash nanoparticles were used for coating as well to investigate their effect on thermal performance. The findings in this study can be used for further research in aerogel-based high-performance materials for thermal protection.
\end{abstract}

Keywords: Kevlar; Aerogel; Thermal Protective Properties; Laser Radiation

\section{Introduction}

Aerogels, prepared by the polymerization of silicic acid or by the aggregation of particles of colloidal silica through a supercritical drying process, are highly porous open cell solid materials that features thermal conductivities as low as $0.013 \mathrm{Wm}^{-1} \mathrm{~K}^{-1}$ [1-3]. Since the silica aerogels comprise highly open structures in which the secondary particles of silica are connected to each other with only few siloxane bonds, the silica aerogels generally have poor mechanical stability, such as low strength and high brittleness. Resulted from the lower production costs of aerogel granules and simple preparation process, existing aerogel particles are usually incorporated with fibres or textile structure to achieve improved thermal performance of the overall structure [4].

It is known that aramid fibers possess lower density, lower thermal conductivity and higher mechanical strength with flexibility, which makes it a good choice for an ever-growing number of applications where a reduction of weight, increase in strength and resistance to corrosion produce

\footnotetext{
${ }^{*}$ Corresponding author.

Email addresses: mohanapriya.venkataraman@tul.cz (Mohanapriya Venkataraman), xiaoman.xiong@tul.cz (Xiaoman Xiong).
} 
significant improvements in safety and efficiency [5]. High-performance fabrics and composites made of aramid fibers are used in Military body armor, firemen's uniforms, protective clothing and motorcyclist's suits. Thermal behavior analysis of these materials under a severe environment, is important. The decomposition temperature of aramid fibers in air reaches up to $450{ }^{\circ} \mathrm{C}$ which is much higher than that of other organic fibers [6]. It can be inferred that the silica aerogel embedded aramid fabrics would be suitable for some higher temperature insulation rather than the ambient temperature only.

Studies on combining silica aerogel with aramid are mainly focusing on composites of aramid fiber reinforced silica aerogel in which the aramid fibers acted as reinforcements. The composites have been prepared by sol-gel method via ambient pressure drying [6]. The heat transfer characteristics of the composites under a transient plane heat source and hot plate experiment revealed that both the aramid fibers and the aerogel matrix contribute to the overall thermal conductivity. Meanwhile, the temperature differences of the composites increased with the fiber content and displayed linearity to some extent. Aramid fibers have been successfully grafted with the mesoporous structure of silica aerogel to reduce the fiber thermal conductivity through a serious process [7]. Thermal conductivity of the as-prepared aramid fiber blanket is $25 \%$ lower than that of the original ones, whereas the thermal stability changes little. A study on Kevlar nanofiber aerogel films fabricated through spin-coating, sol-gel processing and subsequent freeze-drying found that the typical aerogel structure endowed the films with excellent thermal insulation ability to prevent effective heat transfer and reduce the IR radiation [8]. All these studies were focusing on thermal comfort properties of the composites composed by aramid fibers and aerogels, however, thermal protective properties of aerogel-based Kevlar fabric were not well investigated.

The present paper deals with aerogel coated Kevlar fabrics for thermal protection. Kevlar woven fabric coated with aerogel particles were fabricated by using RTV silicone as binding materials. Thermal protection of the prepared materials under high temperature up to several hundred degree was determined with a laser system and thermometer. The effect of aerogel and fly ash nanoparticles on thermal protection were investigated and discussed. Thermal properties of the prepared fabrics under ambient temperature were evaluated by a thermal camera.

\section{Experimental}

\subsection{Materials}

Plain woven fabric of Kevlar 29 with 5.7 ends/cm and 10 picks/cm was selected to carry out coating. The fabric had an areal density of $265.80 \mathrm{~g} / \mathrm{m}^{2}$ and a thickness of $0.562 \mathrm{~mm}$. Silica aerogel granules used were hydrophobic, mesoporous and had nearly $98 \%$ of air and $2 \%$ solid. The specifications are presented in Table 1. Due to the interconnected nanoporous characteristic, aerogel can hold air within its structure and does not allow free flow of air, which enables it to be a superior thermal insulation material. Fly ash class F, the most common coal-fly ashes, was used for coating as well to investigate its effect on thermal properties of the coated fabrics. The fly ash is very fine grained with a fineness between 2 micrometers and 300 micrometers, with a density of $1.34 \mathrm{~g} / \mathrm{cm}^{3}$. Thermosetting resin, Zhermack ZA 13 Mould 45, was used as binder to combine the aerogel and fly ash particles with Kevlar fabrics. ZA 13 MOULD WT 45 is a bi-component addition cured RTV silicone rubber compatible with all plasters, coatings, polyurethane resins, acrylic resins and polyester resins. The chemical and physical characteristics of this RTV silicone 
rubber are illustrated in Table 2.

Table 1: Details of silica aerogels used for coating

\begin{tabular}{ll}
\hline Properties & Value range \\
\hline Particle size $(\mathrm{mm})$ & $0.1-0.7$ \\
Pore diameter $(\mathrm{nm})$ & $\sim 20$ \\
Porosity $(\%)$ & $>90$ \\
Particle density $\left(\mathrm{kg} / \mathrm{m}^{3}\right)$ & About 120 \\
Surface chemistry & Fully hydrophobic \\
Thermal conductivity $(\mathrm{W} / \mathrm{m} \cdot \mathrm{K})$ & $0.012\left(\right.$ at $\left.25^{\circ} \mathrm{C}\right)$ \\
\hline
\end{tabular}

Table 2: Chemical and physical properties of Zhermack ZA 13 Mould 45

\begin{tabular}{ll}
\hline Parameter & Metric system \\
\hline Mixing ratio & $1: 1$ \\
Density & $1.07 \mathrm{~g} / \mathrm{cc}$ \\
Viscosity of the mix pre-catalisation & $4500 \pm 500 \mathrm{cP}$ \\
Setting time at $23^{\circ} \mathrm{C}$ & $2 \mathrm{~h}-3 \mathrm{~h}$ \\
Hardness Shore-A after 24 hours & $13 \pm 2 \mathrm{ShA}$ \\
\hline
\end{tabular}

\subsection{Preparation of Aerogel-coated Kevlar Fabrics}

The silicone was mixed with catalyst with a ratio of 1:1 to form a homogeneous mixture, the selected particles were added into the as-prepared resin and stirred for 5 minutes until the particles were uniformly dispersed. The mixture was then poured on the Kevlar fabric to be coated with a blade, the obtained fabrics were subsequently cured for 24-48 hours at room temperature. Different content of aerogel particles was used to investigate the effect on thermal protection. Fly ash nanoparticles were incorporated as well to examine its effect on thermal behavior. Details of the resultant fabrics are listed in Table 3 [10].

\subsection{Measurements}

\subsubsection{SEM characterization of fly ash nanoparticles}

The surface morphology of fly ash was observed by using TS5130 Vega-Tescan scanning electron microscope (SEM). During the measurement, the electron column was kept at vacuum and the accelerating voltage used was $20 \mathrm{kV}$.

\subsubsection{Laser radiation}

A commercial pulsed $\mathrm{CO}_{2}$ laser (MARCATEX 150 FLEXI, EasyLaser) was used to evaluate thermal performance of the fabrics under laser radiation. The laser system provides a laser beam 
Table 3: Specifications of Kevlar fabrics coated with different particles

\begin{tabular}{cccccccc}
\hline & \multicolumn{2}{c}{ ZA 13} & & & & \\
Sample No. & $\begin{array}{c}\text { Silicone } \\
\mathrm{g} / \mathrm{m}^{2}\end{array}$ & $\begin{array}{c}\text { Catalyst } \\
\mathrm{g} / \mathrm{m}^{2}\end{array}$ & $\begin{array}{c}\text { Aerogel } \\
\mathrm{g} / \mathrm{m}^{2}\end{array}$ & $\begin{array}{c}\text { Fly ash } \\
\mathrm{g} / \mathrm{m}^{2}\end{array}$ & Thickness mm & $\begin{array}{c}\text { Area density } \\
\mathrm{g} / \mathrm{m}^{2}\end{array}$ & $\begin{array}{c}\text { Bulk density } \\
\mathrm{kg} / \mathrm{m}^{3}\end{array}$ \\
\hline A0F0 & 320 & 320 & 0 & 0 & 0.895 & 789.49 & 882.11 \\
A3F0 & 320 & 320 & 3.47 & 0 & 1.230 & 807.11 & 656.19 \\
A8F0 & 320 & 320 & 8.07 & 0 & 1.349 & 807.24 & 598.40 \\
A13F0 & 320 & 320 & 13.89 & 0 & 1.396 & 830.32 & 594.79 \\
A0F27 & 320 & 320 & 0 & 27.78 & 0.886 & 811.55 & 915.97 \\
A8F27 & 320 & 320 & 8.07 & 27.78 & 1.212 & 841.60 & 694.39 \\
AF & 0 & 0 & 0 & 0 & 0.562 & 265.80 & 472.95 \\
\hline
\end{tabular}

of wavelength $10.6 \mu \mathrm{m}$ which interacts with fibers by thermal processing including solid heating, melting, evaporation, and high temperature combustion [11]. Energy consumed during the laser radiation process can be optimized through the optimization of process parameters. Different experimental conditions concerning laser radiation were tested in order to investigate the effect of different treatment parameters on the thermal protection of Kevlar fabrics. The sample to be tested was placed in the testing cabinet with the coated surface facing to the laser beam. A $5 \mathrm{~cm}$ by $5 \mathrm{~cm}$ square opening located in the bottom body of the testing cabinet gives access to the measurements of surface temperature from the backside of the fabric. An IR thermometer connected to the Optris software was fixed under the cabinet to record the fabric temperature through the opening. The setup of the device was shown in Fig. 1.

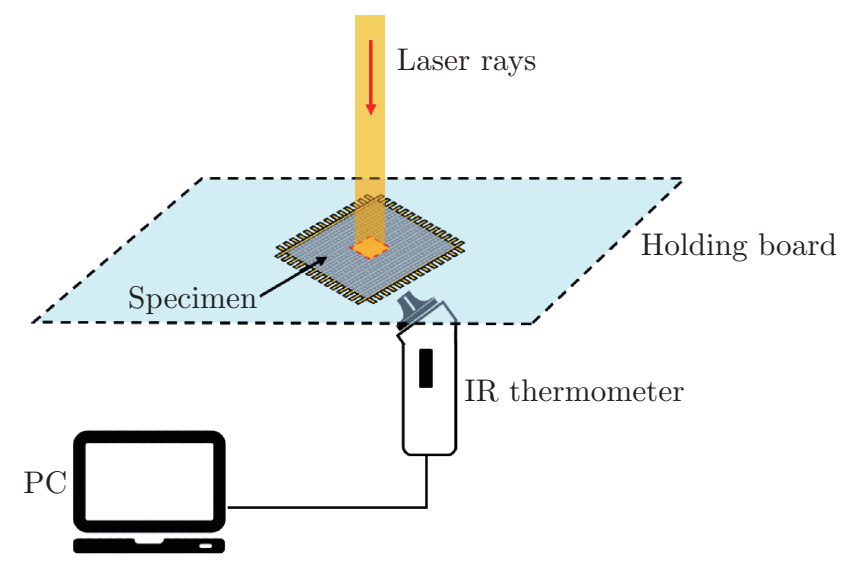

Fig. 1: Setup of the measurement of thermal protection properties

\subsubsection{Infrared thermography}

Thermal images are visual displays of the amount of infrared energy emitted, transmitted, and reflected by an object. The amount of radiation emitted by the heat plate through the fabric was detected. Therefore, the thermography allows to see temperature variations. Infrared thermography measurement was conducted by using thermal camera. A vertical hot plate maintained at constant temperature was used as heat source, the specimen was flatly attached onto the hot 
plate with the help of a frame tool, thermal images were taken by thermal camera at selected distance from the hot plate after different time intervals. The room temperature was kept at $25 \pm 2^{\circ} \mathrm{C}$. A thermal image reveals the amount of radiation emitted by the heat plate through the fabric. The thermal insulation value can be calculated via the following equation

$$
I=\frac{T_{\text {hot plate }}-T_{\text {fabric }}}{T_{\text {hot plate }}-T_{\text {air }}}
$$

where $T_{\text {hot plate }}$ is the hot plate temperature $\left({ }^{\circ} \mathrm{C}\right), T_{\text {fabric }}$ is the fabric surface temperature $\left({ }^{\circ} \mathrm{C}\right)$, $T_{\text {air }}$ is the room temperature $\left({ }^{\circ} \mathrm{C}\right)$.

\section{Results and Discussion}

\subsection{Morphology of Fly Ash Nanoparticles}

The size distribution of fly ash nanoparticles is quite large as shown in Fig. 2, most of the particles were less than 20 micrometers in size. The particles appear to have irregular shapes, the grains have aggregated to form nanoclusters.
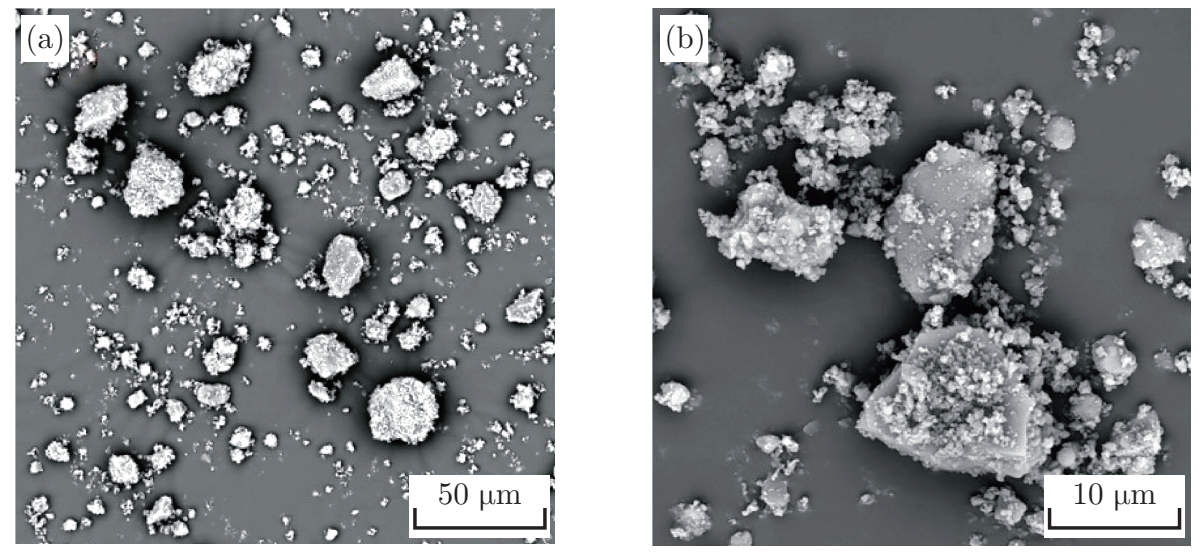

Fig. 2: SEM of fly ash nanoparticles

\subsection{Thermal Protection of Aerogel-coated Kevlar Fabrics under Laser Radiation}

The maximum temperatures of the Kevlar fabric and aerogel coated fabrics for different pixel times on the back surface are shown in Fig. 3. Apparently, the back-side temperature of the uncoated Kevlar fabrics is in the range of $275-530{ }^{\circ} \mathrm{C}$, much higher than the safety margin. But for the coated Kevlar fabrics, the back-side temperature is less than $120{ }^{\circ} \mathrm{C}$, revealing a sufficient protection provided by the coating to block the heat from the laser ray. Especially at laser radiations with pixel time $330 \mu \mathrm{s}$, the surface temperatures of the coated fabrics are $400-440{ }^{\circ} \mathrm{C}$ lower than that of the uncoated fabric, indicating that the aerogels present on the fabric play a vital role on the back-side temperature of the overall structure. As the aerogel content used for coating increases, the fabric temperature decreases as well, a better protection from the laser 
radiations is achieved. It is also found that the fly ash nanoparticles contribute to slightly lower back-side temperature and the combination of aerogels with fly ash nanoparticles has positive effect on thermal protective properties.

The pixel time is the time in microseconds used to mark each pixel of the image. The higher the pixel time, the more heat energy provided by the laser beam and the higher the generated temperature on the coated surface of the fabrics. The pixel time used for laser radiation influences the back-surface temperature as well. The Kevlar fabric and coated fabrics exhibit increased backsurface temperature as the pixel time increases. The linear fittings for the samples found that the correlation coefficients are all above 0.90 as seen in Fig. 3, meaning that the temperature from back side of the fabrics are directly proportional to the pixel time.
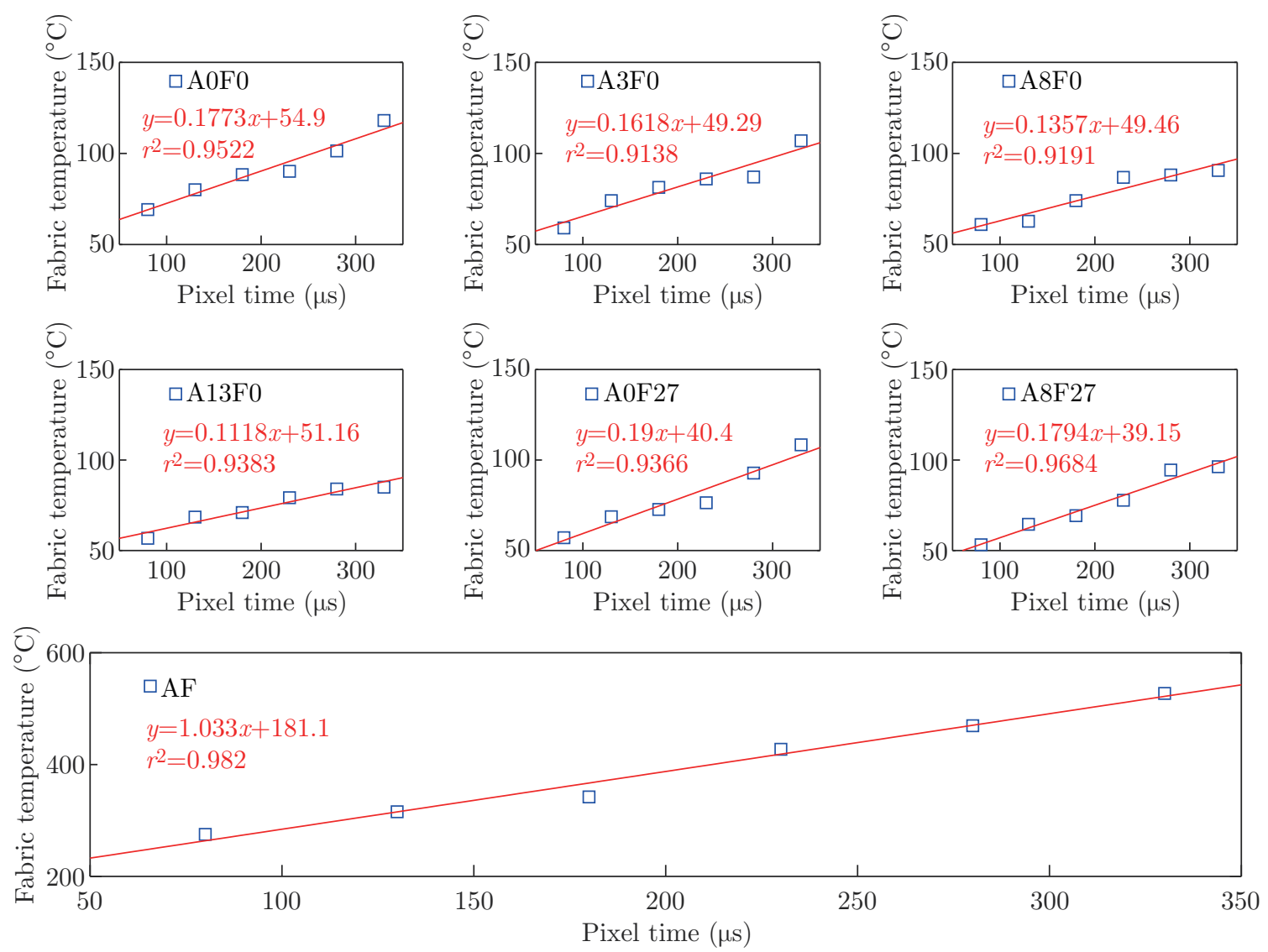

Fig. 3: Effect of pixel time on fabric temperature

Fig. 4 shows clearly the effect of aerogel content on back-surface temperature of the coated Kevlar fabrics under laser radiation. The temperature of the coated Kevlar fabric decreases with the increase in aerogel content, and this trend becomes more obvious when the pixel time for laser radiation is increased. The analysis of linear fit model found that the temperature of the coated Kevlar fabric decreases linearly with the increase in aerogel content. Meanwhile, it could be observed that the higher the pixel time, the faster the back-side temperature drops with the increasing in aerogel content. This is because the aerogel functions as a barrier to block heat transfer by means of holding the air molecules inside its interconnected nano-structural network. As a result, as the pixel time increases the deceleration of the air molecules movement caused by the aerogel was enhanced, and this became a dominant factor in determining thermal protective properties of the overall structure, leading to much lower heat transferred to the back-side of the 

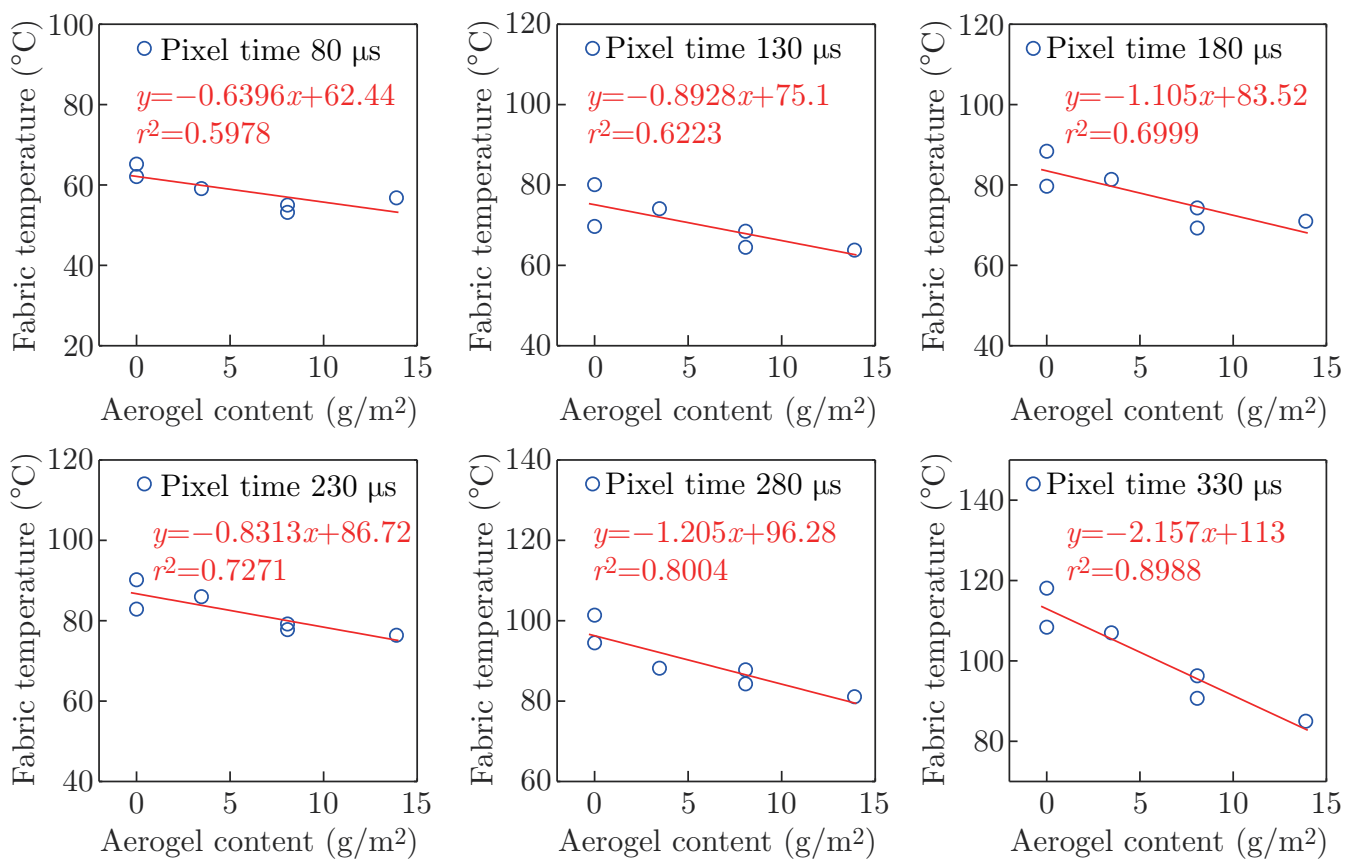

Fig. 4: Dependence of fabric temperature on aerogel content

coated fabric with higher aerogel content. It can be concluded that the Kevlar fabrics coated with silica aerogel provides good thermal protection under high temperature.

\subsection{Thermal Insulation under Ambient Temperature}

The accuracy of thermography is still under discussion because environmental conditions, such as ambient temperature and airflow, may influence surface temperatures in regions of interest and may hamper the interpretation of images [12]. It has been described that a change in infrared camera distance from a subject influences the results because a thermographic image taken from a greater distance may result in a smaller region of interest with fewer pixels and lower resolution. In this study, sample A13F0 and AF were selected to conduct measurements with different distances from thermal camera. As seen in Fig. 5, the fabric to camera distance has insignificant effect on
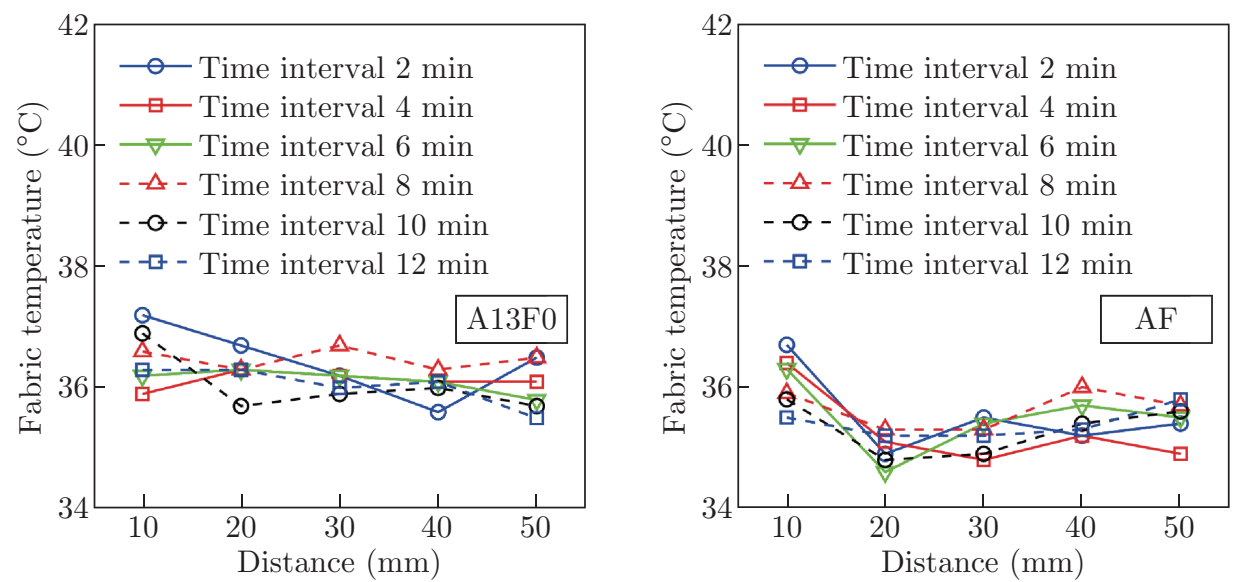

Fig. 5: Effect of fabric to thermal camera distance on the measured fabric temperature 
the recorded temperature of the fabric surface especially when this distance is over $20 \mathrm{~cm}$.

The recorded surface temperature with fabric to camera distance $40 \mathrm{~cm}$ slightly varies with the composition of the coatings. According to the recorded temperatures, thermal insulation values of different fabrics are calculated and compared in Fig. 6 [10]. The coated Kevlar fabrics has much lower porosity and fabric thickness, and thus the samples demonstrate low thermal insulation values ranging from 0.168 to 0.256 . Meanwhile, as the aerogel content increases, the coated fabric shows an increasing thermal insulation value, which further confirmed that the aerogel plays a role in improving thermal insulation at low temperature. The addition of fly ash nanoparticles lead to a slightly higher thermal insulation value, this could be attributed to its high surface area and thus more absorption of heat energy.

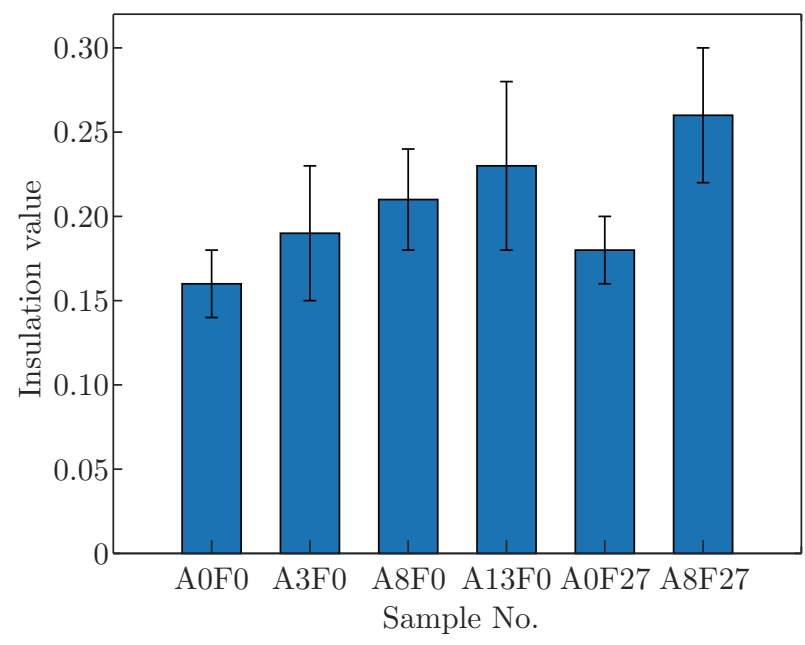

Fig. 6: Comparison of thermal insulation values from different fabrics

\section{Conclusion}

Kevlar woven fabrics coated with aerogel particles were fabricated via blade coating with silicone as binding materials. Fly ash nanoparticles were incorporated as well. Thermal behavior of the prepared fabrics under both ambient temperature and high temperature up to several hundred degree were evaluated and discussed. The effect of aerogel and fly ash nanoparticles on thermal protection were investigated and analyzed. Results showed that at laser radiations with pixel time $330 \mu \mathrm{s}$, the back-side temperatures of the aerogel-coated Kevlar fabrics are $400-440{ }^{\circ} \mathrm{C}$ lower than that of the uncoated fabric, indicating a good thermal protection provided by the coating to block the heat from the laser ray. By increasing the aerogel content, a better thermal protection is achieved. Meanwhile, the temperature from back side of the fabrics are directly proportional to pixel time, the back-side temperature of the coated Kevlar fabric decreases linearly with the increase in aerogel content. It can be concluded that the Kevlar fabrics coated with silica aerogel provides excellent thermal protection under high temperature. Results of thermography under ambient temperature reveals that as the aerogel content increases the coated fabrics demonstrated improved thermal insulation ability. Moreover, the addition of fly ash nanoparticles could contribute to a slightly higher thermal insulation value as well. 


\section{Acknowledgement}

This work was supported by the Ministry of Education, Youth and Sports of the Czech Republic and the European Union - European Structural and Investment Funds in the frames of Operational Programme Research, Development and Education - project Hybrid Materials for Hierarchical Structures (HyHi, Reg. No. CZ.02.1.01/0.0/0.0/16_019/0000843), project "Modular platform for autonomous chassis of specialized electric vehicles for freight and equipment transportation", Reg. No. CZ.02.1.01/0.0/0.0/16_025/0007293, project "Design optimization and application of smart heat-insulating nano-layers" [LTACH-17014, 18301] and project "Intelligent thermoregulatory fibers and functional textile coatings based on temperature resistant encapsulated PCM" SMARTTHERM (Project No. TF06000048).

\section{References}

[1] Ller RK. The Chemistry of Silica-solubility, polymerization, colloid and surface properties, and biochemistry. Angewandte Chemie 1980; 92(4): 328.

[2] Valdes-Solis T and Fuertes AB. High-surface area inorganic compounds prepared by nanocasting techniques. Materials Research Bulletin 2006; 41(12): 2187-2197.

[3] Hoseini A, McCague C, Andisheh-Tadbir M, Bahrami M. Aerogel blankets: From mathematical modeling to material characterization and experimental analysis. International Journal of Heat and Mass Transfer 2016; 93: 1124-1131.

[4] Sanz-Pont D, Sanz-Arauz D, Bedoya-Frutos C, Flatt RJ and López-Andrés S. Anhydrite/aerogel composites for thermal insulation. Materials and Structures 2016; 49(9): 3647-3661.

[5] Kabir RB, Ferdous N. Kevlar-the super tough fiber. International Journal of Textile Science 2012; 1(6): 78-83.

[6] Li Z, Gong L, Cheng X. Flexible silica aerogel composites strengthened with aramid fibers and their thermal behaviour. Materials \& Design 2016; 99: 349-355.

[7] He S, Sun G, Cheng XD. Nanoporous SiO2 grafted aramid fibers with low thermal conductivity. Composites Science and Technology 2017; 146: 91-98.

[8] Lyu J, Liu ZW, Wu XH. Nanofibrous Kevlar aerogel film and their phase-change composites for highly efficient infrared stealth. ACS Nano 2019; 13: 2236-2245.

[9] Dolezal I, Hes L and Bal K. A non-destructive single plate method for measurement of thermal resistance of polymer sheets and fabrics. International Journal of Occupational Safety and Ergonomics 2018.

[10] Venkataraman M, Xiong X, Yang T, Yang K, Wang YF, Mishra R and Militký J. Thermal performance of Kevlar woven fabrics coated with silica aerogel. TBIS Proceeding, 2019.

[11] Sulaiman FA, Yilbas BS, Karakas FC, Ahsan M, Mokheimer EMA. Laser hole cutting in Kevlar: modeling and quality assessment. The International Journal of Advanced Manufacturing Technology 2008; 38: 1125 .

[12] Simone W, Heinz HFB, Johannes S, Alexander T, Christian S. Effects of infrared camera angle and distance on measurement and reproducibility of thermographically determined temperatures of the distolateral aspects of the forelimbs in horses. Journal of the American Veterinary Medical Association 2013; 242: 388-395. 